\title{
A Flat Routing Protocol for Sensor Networks
}

\author{
Anita Kanavalli ${ }^{1}$, Dennis Sserubiri ${ }^{2}$, P Deepa Shenoy ${ }^{3}$, Venugopal K R4 and L M Patnaik ${ }^{5}$ \\ ${ }^{1,2,4}$ Department of Computer Science and Engineering, University Visvesvaraya College of Engineering Bangalore \\ ${ }^{5}$ Vice Chancellor, Defence Institute of Advanced Technology (Deemed University), Pune India \\ e-mail:1 anita.kanavalli@gmail.com
}

\begin{abstract}
The way in which Wireless Sensor Networks (WSN) are designed requires that energy be taken as the most crucial element if WSNs are to be used in the most effective way to serve the purpose for which they have been deployed in the target region. Routing protocols are the main aids that can assist in reducing the energy consumption required by the transmission of data throughout the sensor networks. In this paper we have proposed a flat routing protocol for sensor networks. This approach is one of the simplest protocols in terms of the route determination process and the number of messages through the network. Routes from the source to the destination are determined by use of only the hop count and remaining energy of the neighbor nodes for each node. The proposed protocol is simulated and is compared with the flood routing protocol.
\end{abstract}

Keywords- wireless sensor networks, source initiated, energy consumption, node life time, flat routing

\section{INTRODUCTION}

E ver since the emergence of wireless sensor networks (WSNs) about two decades ago a lot of research regarding routing protocols in WSNs has been done. A wireless sensor network is a network of sensor nodes deployed in urban or remote areas to monitor on going events as they happen. Wireless sensor network can be used for military, health, environmental, industrial purposes and so on. The sensor nodes can be deployed in large numbers ranging from tens to thousands in the area of interest. These are usually small, inexpensive, low power sensors that can be deployed anywhere. Sensor nodes are meant to be self configuring having one or more embedded sensors, wireless communication and data processing units. Sensor nodes are characterized by their limited supply of energy.

Due to the limited battery capabilities, sensor nodes suffer an energy resource constraint such that if there happens to be a failure of even a single node, the whole network topology changes requiring the network setup to be initiated again. This means that the life time of the network greatly depends on the life time of the individual nodes in the network of sensor nodes. Since sensor nodes are deployed to monitor events happening in a particular region, there is always a sink node or base stations to which the sensing nodes have to route the sensed data for processing. The main advantage of the sink node is its prolonged energy supply since it is always accessible and therefore it always guarantees that if sensed data reaches the sink node, then the probability of loosing the sensed data is reduced which is not the case for the source nodes. Node failure can be due to battery outage since sensor nodes are equipped with a fixed source of battery power. An important factor that influences the consumption of more power in wireless sensor network is that each sensor node consumes power not only for sensing but also for processing the sensed data and transmitting or receiving data to or from its neighbors. These are the reasons for which the efficient use of power is the primary and perhaps the most important consideration for designing a wireless sensor network protocol [1]. Routing protocols in wireless sensor network are designed in such a way that they keep the network life time for a longer period by efficiently utilizing the available resources in the most economical way.

Routing in wireless sensor network is a challenging task because of their characteristics that differentiate them from other wireless networks. Usually the number of nodes in the network is so high that it is not possible to have a global addressing scheme as the overhead would be very high to maintain. In WSNs, sometimes getting the data is more important than knowing the node identifiers (IDs) of the nodes that sent the data. In contrast to typical communication networks, almost all applications of sensor networks require the flow of sensed data from multiple sources to a particular base station. This however, does not prevent the flow of data to be in other forms like multicast or peer to peer. The sensor nodes are tightly constrained in terms of energy processing, and storage capacities. Thus, they require careful resource management. In most application scenarios, nodes in wireless sensor networks are generally stationary after deployment except for a few mobile nodes. A number of factors do influence the design of routing protocols for wireless sensor network. They are node deployment, energy consumption, scalability, data aggregation, coverage and connectivity. There exist a number of routing protocols classified according to network structure. They are hierarchical routing protocols, flat routing protocols and Location based routing protocols. In flat routing protocols, all nodes in the sensor network have equal roles in gathering information [2]. They all have the same information about the state of the network. Some of the flat routing protocols that have been proposed include: Directed Diffusion, Spin, Rumor routing, Minimum Cost 
Forwarding Algorithm, Gradient Based, Cougar, and Acquire. In this paper a flat routing protocol has been proposed.

Motivation: Proposing a new routing protocol for wireless sensor networks is one of the most challenging works to accomplish successfully. Despite the challenges, it is crucial that all important issues be addressed in order to come up with the best results.

Contribution: In this paper, a new flat routing protocol is proposed and simulated. The issues that are addressed are the calculations of the energy values using the radio model in [3] with regard to the distances between nodes. This is mainly due to the fact that when nodes are transmitting data diagonally, more energy is used for transmission and reception of messages than when transmitting or receiving data from vertical or horizontal neighbors. In a uniform network, the nodes are able to communicate diagonally. Then the distance between the diagonal neighbors cannot be the same as those for horizontal or vertical neighbors. It will always be much larger. Also during network maintenance, it is possible that nodes remove dead neighbors from their table during the energy updates process rather than wait for the periodic network maintenance message.

Organization: The rest of the paper is organized as follows: Section II gives overview of related work. The model and the problem definition, the analysis of the algorithm is presented in section III. The implementation details and the performance analysis are discussed in section IV. Section V contains the conclusion.

\section{RELATED WORK}

C Intanagonwiwat et al [4] proposed one of the most popular routing protocols for wireless sensor network called directed diffusion. This is data centric and application aware routing protocol. All data generated is named with attribute-value pairs. Data that is on its way to the sink is combined as it is forwarded in order to remove redundancy, minimizing the number of transmissions thus saving battery energy which in turn prolongs the network life time. The performance of data aggregation methods in directed diffusion methods is affected by factors such as position of the source nodes, number of sources and the network topology [2].

SPIN is a family of adaptive flat routing protocols that were proposed by Heinzelman et al [5],and [6] that use a technique of data negotiation and resource adaptive algorithms. These families of protocols disseminate information to each and every node in the network with the assumption that all nodes in the wireless sensor network could be potential base sinks. This enables a user to request for information from any node in the network and get the requested information since all the nodes in the network have the same information. In these protocols all neighbor nodes have the same data and it is only data that the others nodes do not have that distributed to the neighbor nodes.
Rumor routing [7] is intended to be used in areas where geographical routing cannot be used. It varies from directed diffusion in a sense that when the number of events is small and the requests are large, the idea is to flood the events. Rather than flooding the entire network with queries, the queries are routed to only the nodes that have observed events. In order to route events through the network, the rumor routing algorithm employs long-lived packets, called agents. When a node detects an event, it adds such event to its local table, called events table, and generates an agent [2]. These agents eventually disseminate information to distant nodes about the state of local events. In rumor routing, if a node generates a request for an event, the other nodes which know the route may generate a response to the request by inspecting their events table. This eliminates the need for flooding the whole network in turn reduces communication costs. Tests show that rumor routing is good at energy saving compared to flood routing.

In paper [8] the main objective is to increase the network life time as its name suggests. Energy aware routing is a destination initiated reactive routing protocol that maintains a set of paths to the base station. This is a possible means of a probability value that depends on how low the energy consumption of each node can be attained. By choosing different paths at different times, it is possible to conserve the energy of the individual nodes without depleting them quickly. Through localized flooding, it is possible to discover all routes from source to destination and their costs.

SEER: A Simple Energy Efficient Routing Protocol for Sensor Networks [9] was proposed by Gerhard P. Hancke, C. Jaco Leuschner as an energy efficient routing protocol for sensor networks. It is a flat routing protocol that achieves energy efficiency by use of hop count, remaining energy in the nodes and routing decisions are based on the distance to the base station. These metrics are used to determine the routes for forwarding data to the sink. It is a source initiated routing protocol and it uses a uniform network to achieve this efficiency. The authors of this protocol claim that if the sink node it at the center of the network with the source nodes uniformly distributed from the sink and from each other, it is possible that significant energy efficiency can be achieved.

\section{MODEL AND ALGORITHM}

\section{A. Radio Model}

There is a great deal of research in the area of lowenergy radios. The assumptions about the energy dissipation in the transmit and receive modes, will change the advantages of different protocols. The proposed protocol operation uses Heinzelman et al [2] radio model for the energy consumptions of the messages. We assume that radio dissipates $n \mathrm{~J} / \mathrm{bit}$ to run the transmitter and the receiver circuit and energy is dissipated by the transmitter amplifier. We also assume 
that there is an energy loss due to channel transmission. According to this radio model the energy required for the transmission and reception of the messages is given by the following equations.

$E_{T x}=E_{E l e c} \cdot k+\varepsilon_{a m p} \cdot k \cdot d^{2}$ where, $\quad E_{T x}$ is the energy required for the transmission of the messages. $E_{R x}=E_{E l e c} . k$ where, $E_{R x}$ is energy required for reception of messages, and $E_{E l e c}$ is the energy consumed by the transceiver electronics, $k$ is the size of the message in bits, $\varepsilon_{a m p}$ is the energy consumed by the transmitter amplifier and $d$ is the transmission distance in meters. For these parameter values, transmitting and receiving a message is not a low cost operation. Thus the protocol should reduce the transmit distances and also the number of transmit and receive operations for each message. The radio channel is symmetric such that the energy required to transmit a message from node $A$ to node $B$ is the same as the energy required to transmit a message from node $B$ to node $A$. All sensors are sensing the environment at a fixed rate and thus always have data to send to the enduser.

\section{B. Problem definition}

The protocol requires five steps for its operation.

- $\quad$ Network setup and neighbor discovery

- Transmitting new data

- Forwarding data

- $\quad$ Energy updates

- Network maintenance.

The issues that are addressed are the calculation of energies made using the radio model in [11] with regard to the distances between nodes and removal of dead nodes from the neighbor table.

During network maintenance, it is possible that nodes remove dead neighbors from their table during the energy updates process rather than wait for the periodic network maintenance message.

\section{Algorithm}

The algorithm for the proposed protocol is explained in this section

1. In the network setup and neighbor discovery, the sink node broadcasts a network setup message to its neighbors which in turn forward it to other neighboring nodes. This message contains network setup information that will be updated as the message is forwarded. The information includes the hop count and energy, which change as the message is forwarded further each node storing this information about its neighbor and forwarding it.

2. During the transmission of new data, if a node has information to be transmitted, the node checks in its routing table for the best neighbor that is close to the sink based on the hop count and energy of the node. This is done starting with the hop count and then the energy depending on whether the node has more than one node in its neighbor table with the same hop count and then which has the highest energy remaining.

3. During the data forwarding process, the same process for neighbor node selection is done except that the creator node cannot be selected this time during the neighbor selection process. When the node's remaining energy falls below a certain threshold, the node transmits an energy update message to its neighbors informing them about its energy status. When the nodes receive the energy message, they remove the node that sent the message from their neighbor table.

4. During the network maintenance, the sink periodically sends a network maintenance messages for the nodes to add new neighbors that have joined the network.

\section{The Mathematical model}

1. The distances between the nodes are assumed to be uniform including the diagonal neighbors of the nodes. This is not the case during the calculation of the distances of the diagonal members, Pythagoras theorem is used in order to determine the distance in the energy calculation of the power dissipated in transmission and the reception of the messages. Taking uniform distances will yield wrong results as the distance from the source to the next diagonal neighbor increases. Though during the protocol operation, it is indicated that the neighbors have to be a meter apart. Assuming the distances are more than a meter a part, then the diagonal distances will be much bigger.

It is possible that the distances be calculated using the channel node Free Space Loss Model:

$$
P_{r}=\frac{P_{t}}{\left(\frac{4 \pi}{\lambda}\right)^{2} * d^{2}}
$$

where $P_{r}$ is the received power, $P_{t}$ the transmitted power, $\lambda$ is the wavelength of the signal and $d$ is the distance between sender and receiver.

2. During the network maintenance step, it is mentioned that the sink sends a network maintenance message for the nodes to remove neighbors that have failed. This process can be done during the energy update step when nodes transmit energy update messages to their neighbor. This reduces the amount of energy that will be used during the storage of information for failed nodes. The reception and the transmission energy is computed using the radio model formula in section III $\mathrm{A}$ where $E_{\text {Elec }}=$ $50 \mathrm{~nJ} / \mathrm{bit}$ and $\varepsilon_{\text {amp }}$ is $100 \mathrm{pJ} / \mathrm{bit}$. Node energy is reduced by for Reception and Transmission of messages.

\section{IMPLEMENTATION AND PERFORMANCE ANALYSIS}

\section{A. Implementation}

OMNET [14] is a discrete event simulator uses both network description language and $\mathrm{C}++$ to define all the objects in the simulation. In this case objects included 
things such as the messages and nodes. The messages are defined with the various necessary fields for the network layer. The proposed algorithm was defined using $\mathrm{C}$ and inbuilt functions of OMNET. The node details are as follows. Before the network and neighbor discovery process, node were assigned initial parameters which included

- Node address

- Initial energy $=5 \mathrm{~mJ}$

- $\quad$ Minimum energy $=0.00475 \mathrm{~J}$

- $\quad$ Hop count $=0$

The neighbor table entry in each node had the information of address, energy and hop count. There are three types of messages used in the simulation.

1. The network setup and neighbor discovery message, the parameters included are source address, destination address, hop count, energy level of sender node and the sequence number.

2. Data message, the parameters included are source address, destination address, creator address, energy level of sender, payload, and hop count.

3. Energy message, the parameters included are source address, destination address, energy level, and hop count

The network setup and neighbor discovery message was set to 64bits, data message was setup to 105 bits and energy message to 56 bits. The first message to be sent through the network is the network and neighbor discovery message broadcast by the sink node. As the message is routed through out the network, nodes kept updating their neighbor tables to include newly discovered neighbors that are directly connected to them and also node parameters, i.e. the hop count of the node, energy were updated to reflect the reduction in energy, hop count and address read from the received network setup and neighbor discovery message. Nodes also updated their node parameters to reflect their reduction in initial energy and hop count. As each node received this message, node parameters were updated and messages parameters where changed before forwarding the message to the next neighbor. After all the nodes in the networks had updated their parameters, then a data messages is generated.

Generated data messages which are generated by nodes are routed through the networks following the algorithm in section IV A. Also the node energy of the node that generated a data message is updated to reflect the reduction in energy before sending out the messages and neighbor table energy for the node selected as the forwarding node was updated in the neighbor table. Only the creator address did not change until the message was received the sink node. If the node's energy got below the set minimum energy, then this message broadcast out a message to its neighbors to inform them of its status. Every node that received this message removed this node from it neighbor table since it has failed. The algorithm for the neighbor selection explained here below.

1. If neighbors hop count $<$ own hop count

2. If there is only one such neighbor go to 3 else go to 4
3. Select neighbor if energy is above predefined threshold, go to 5

4. Select neighbor with highest remaining energy

5. Update neighbors remaining energy table

6. Send the message and end

7. If neighbors hop count $=$ own hop count go to step 2

8. Discard the message and end

We have implemented flood routing protocol and the proposed protocol in OMNET.

\section{B. Simulation Setup}

The simulation setup was a network of 49 nodes uniformly distributed which were equidistant from each other. Each node had an address which was its index in the array of nodes and parameters for energy, sequence number and the address. The figure 1 shows the node setup in OMNET considered for the simulation of both the protocols.

Step one of implementing the proposed protocol is, setting up the network as a uniform network with the sink node being in the center. The simulation begins with the broadcast message sent by the sink node to setup the network by updating the hop count, address and energy levels of each node in the network. Each node stores the details of each of its 8 neighbors. As each node receives the broadcast message, it reads the hop count of the sender and energy level of the sender, stores these values in its neighbor table for that sender that sent the message and then changes the source address to its own and energy, increments the hop count and then forwards the broadcast message.

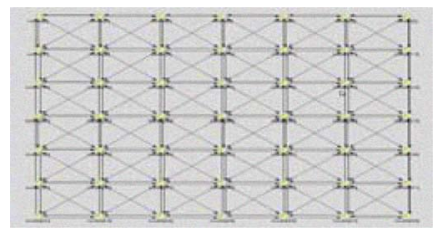

Fig. 1: The nodes considered for the simulation

Before sending the message, the node reduces its energy by the number of messages it received and the number of messages it sent out. After the network has been successfully setup with each node having up to date information of its neighbors and its own parameters then a data message is generated by any node in the network. For each data message, the creator node will reduce its own energy as the energy is spent in transmission, and then sends out the message with its own source address, creator address and energy level. The selected neighbor's energy will have to be updated in the source node before sending out the data message. When a node receives the data messages, it reads the source node address, updates its energy value in its neighbor table, reduces its own energy by the Reception Energy value, and repeats the same procedure as the source node for selecting neighbor according to the neighbor selection algorithm as explained in the previous section. Then the node forwards the message. 
The data message will eventually reach the sink node. When a nodes' energy fall below a certain threshold, in this case $0.0475 \mathrm{~J}$, the node will broadcast an energy update message. The node will reduce its own energy by the transmission energy value. The minimum energy was set to $0.0475 \mathrm{~J}$. Any other messages received will be discarded by that node that is out of energy.

\section{Results}

1. It is realized that flood routing required only 2 data messages to be sent through the network for a single node failure while for the proposed protocol it was 45 messages sent in a network of 49 nodes. This is as shown in the figure 2.

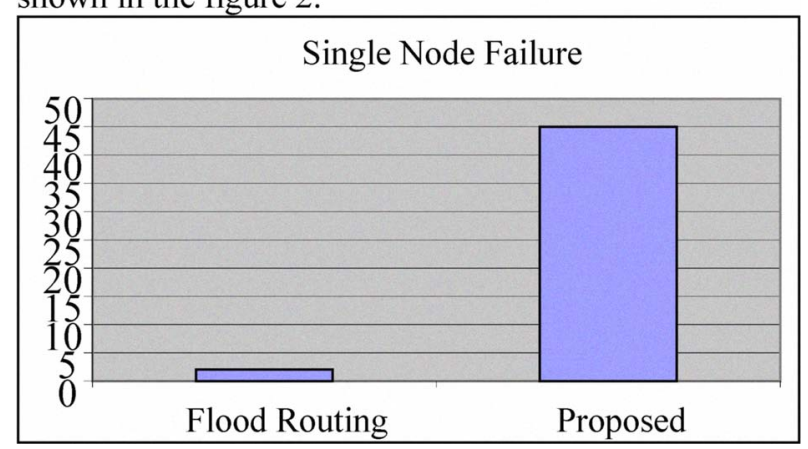

Fig. 2: No. of Messages Vs Single Node Failure

2. The average energy in the network of 49 nodes is measured for the proposed protocol after sending 8 messages and also for the flood routing protocol after sending only 2 messages. The Minimum and the Maximum average energy consumption by the network of 49 nodes is shown in table I.

\section{TABLE I ENERGY CONSUMPTION BY NODES}

\begin{tabular}{|c|c|c|}
\hline & \multicolumn{2}{|c|}{ Energy Consumption in joules } \\
\hline \multirow{2}{*}{$\begin{array}{l}\text { Flood } \\
\text { Routing }\end{array}$} & min avg energy & 0.004878535 \\
\hline & max avg energy & 0.005 \\
\hline \multirow{2}{*}{$\begin{array}{l}\text { Proposed } \\
\text { Protocol }\end{array}$} & min avg energy & 0.00491596 \\
\hline & max avg energy & 0.005 \\
\hline
\end{tabular}

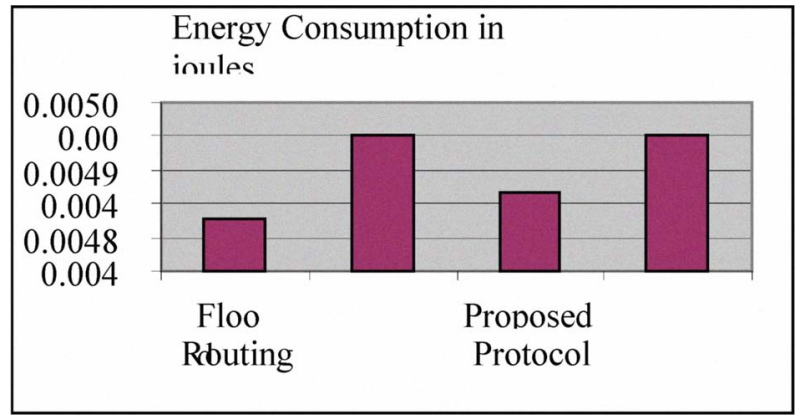

Fig. 3: Average remaining energy for nodes

It is realized that the average remaining energy in the network after sending out data messages was higher in the proposed protocol as compared to flood routing as shown in figure 3 above. In flood routing, the average remaining energy is $0.004878535 \mathrm{~J}$ for only 2 data messages, while for the proposed protocol the energy is $0.00491596 \mathrm{~J}$ for 8 data messages.

\section{CONCLUSION}

It can be therefore realized that a new flat routing protocol for sensor networks that performs far much better than flood routing protocol. This can be visualized in the two graphs. This is evidenced both in the failure of nodes and the average remaining energy of nodes after transmitting data messages in the network. The results confirm that the proposed protocol scales well and improves network lifetime by limiting the number of messages that are sent through the network. Overall, the proposed flat routing protocol achieves a very high level of energy efficiency.

\section{REFERENCES}

[1] F. Zabin, S. Misra, I. Woungang, H.F. Rashvand, N.-W. Ma, M. Ahsan Ali, "REEP: data-centric, energy-efficient and reliable routing protocol for wireless sensor networks", IET Communications, 2008, Vol. 2, No. 8, pp. 995-1008

[2] Jamal N. Al-Karaki, Ahmed E. Kamal, "Routing Techniques in Wireless Sensor Networks: A Survey", Dept. of Electrical and Computer Engineering, Iowa State University, Ames, Iowa 50011

[3] W. Heinzelman, A. Chandrakasan and H. Balakrishnan. "Energy-Efficient Communication Protocol for Wireless Microsensor Networks", Proceedings of the 33rd Hawaii International Conference on System Sciences (HICSS), pp. 1-10. 4-7 January 2000.

[4] Intanagonwiwat, C., Govindan, R. \& Estrin,D, “ Directed diffusion for wireless sensor networks.", IEEE/ACM Transactions Networking, Vol 11., (2003) 2-16

[5] W. Heinzelman, J. Kulik, and H. Balakrishnan, "Adaptive Protocols for Information Dissemination in Wireless Sensor Networks", Proc. 5th ACM/IEEE Mobicom Conference (MobiCom '99), Seattle, WA, August, 1999. pp. 174-85.

[6] J. Kulik, W. R. Heinzelman, and H. Balakrishnan, "Negotiationbased protocols for disseminating information in wireless sensor networks", Wireless Networks, Volume: 8, pp. 169-185, 2002.

[7] Akyildiz, W. Su, Y. Sankarasubramaniam, and E. Cayirci, "A survey on sensor networks", IEEE Communications Magazine, Volume: 40 Issue: 8, pp.102-114, August 2002.

[8] R. C. Shah and J. Rabaey, "Energy Aware Routing for Low Energy Ad Hoc Sensor Networks", IEEE Wireless Communications and Networking Conference (WCNC), March 17-21, 2002, Orlando, FL.

[9] Gerhard P. Hancke, C. Jaco Leuschner. 'SEER: A Simple Energy Efficient Routing Protocol for Wireless Sensor Networks", Department of Electrical and Computer Engineering, University of Pretoria, Lynnwood Road, Pretoria,0002. Reviewed Article--SACJ No. 39, 2007

[10] F. Ye, A. Chen, S. Liu, L. Zhang, "A scalable solution to minimum cost forwarding in large sensor networks", Proceedings of the tenth International Conference on Computer Communications and Networks (ICCCN), pp. 304-309, 2001.

[11] C. Schurgers and M.B. Srivastava, "Energy efficient routing in wireless sensor networks", MILCOM Proceedings on Communications for Network-Centric Operations: Creating the Information Force, McLean, VA, 2001.

[12] Y. Yao and J. Gehrke, "The cougar approach to in-network query processing in sensor networks", SIGMOD Record, September 2002.

[13] N. Sadagopan et al., "The ACQUIRE mechanism for efficient querying in sensor networks" , Proceedings of the FirstInternational Workshop on Sensor Network Protocol and Applications, Anchorage, Alaska, May 2003.

[14] OMNeTT++ "Discreet Event Simulation System" http://omnetpp.org/ 